HEYDE, C. C.

1966

CAN. J. MATH.

Vol. 18, pp. 656-665

\title{
SOME RESULTS ON SMALL-DEVIATION PROBABILITY CONVERGENCE RATES FOR SUMS OF INDEPENDENT RANDOM VARIABLES
}

\author{
C. C. HeYde
}

\section{Reprinted from}

The Canadian Journal of Mathematics 


\title{
SOME RESULTS ON SMALL-DEVIATION PROBABILITY GONVERGENCE RATES FOR SUMS OF INDEPENDENT RANDOM VARIABLES
}

\author{
C. C. HEYDE
}

1. Introduction. Let $\left\{X_{j}, j=1,2,3, \ldots\right\}$ be a sequence of independent, non-degenerate random variables and write

$$
S_{n}=\sum_{j=1}^{n} X_{j}
$$

Under quite a diverse variety of conditions we may obtain

$$
\operatorname{Pr}\left(S_{n}<n^{p} x\right) \rightarrow \frac{1}{2}
$$

as $n \rightarrow \infty$ for all $x,-\infty<x<\infty$, and some real $p \geqslant 0$. For example, suppose the $\left\{X_{j}\right\}$ happen to be distributed identically and belong to the domain of normal attraction of a symmetric stable law with characteristic exponent $\alpha$, $0<\alpha \leqslant 2, \alpha \neq 1$. If $E X_{j}=0$ whenever $E\left|X_{j}\right|<\infty$, then

$$
\operatorname{Pr}\left(S_{n}<n^{p} x\right) \rightarrow \frac{1}{2}
$$

as $n \rightarrow \infty$ for all $x,-\infty<x<\infty$, as long as $0 \leqslant p<1 / \alpha$, in view of the central limit theory. It is the purpose of this paper to establish some results on the rate of convergence of $\operatorname{Pr}\left(S_{n}<n^{p} x\right)$ to $\frac{1}{2}$ by investigating convergence properties of the series

$$
\sum_{n=1}^{\infty} n^{-\beta}\left|\operatorname{Pr}\left(S_{n}<n^{p} x\right)-\frac{1}{2}\right|, \quad-\infty<x<\infty, \beta \leqslant 1 .
$$

Problems of this type could usefully be called small-deviation problems (following terminology that appears to have been introduced by Borovkov 2).

This work is another link in the chain of development of a problem that arose in connection with the study of certain random walks. These previous investigations were restricted to the case of identically distributed random variables. In the random walk context, Spitzer (8) showed that if $E X^{2}<\infty$ and $E X=0$, then

$$
\sum_{n=1}^{\infty} n^{-1}\left(\operatorname{Pr}\left(S_{n}<0\right)-\frac{1}{2}\right)<\infty .
$$

Rosén (7) showed that this series is absolutely convergent, his proof being based essentially on his rediscovery of Gil-Pelaez' (3) inversion formula. Baum

Received May 12, 1965. 
and Katz (1) sharpened the methods of (7) and showed that if $E|X|^{2+\beta}<\infty$ for some $0 \leqslant \beta<1$ and $E X=0$, then

$$
\sum n^{-1+\frac{1}{2} \beta}\left|\operatorname{Pr}\left(S_{n}<0\right)-\frac{1}{2}\right|<\infty .
$$

We go on to put all this theory in a broader context from two points of view. First, we generalize to certain broad classes of non-identically distributed random variables and secondly we include the case $\operatorname{Pr}\left(S_{n}<n^{p} x\right)$ for $p \geqslant 0$, $-\infty<x<\infty$.

2. Preliminaries. Suppose $\left\{X_{j}, j=1,2,3, \ldots\right\}$ is a sequence of independent random variables with corresponding characteristic functions

$$
\left\{\phi_{j}(t), j=1,2,3, \ldots\right\} \text {. }
$$

If there exists a non-degenerate random variable with characteristic function $\phi(t)$ and a constant $\delta>0$ such that

$$
\max _{j}\left|\phi_{j}(t)\right| \leqslant|\phi(t)| \quad \text { for }|t| \leqslant \delta,
$$

we shall say that the sequence $\left\{X_{j}, j=1,2,3, \ldots\right\}$ satisfies the condition $L$. If, in addition, there exists a characteristic function with imaginary part $I(t)$ such that if $I_{j}(t)$ is the imaginary part of $\phi_{j}(t), j=1,2,3, \ldots$, there is a constant $\eta>0$ such that

$$
\max _{j}\left|I_{j}(t)\right| \leqslant|I(t)| \quad \text { for }|t| \leqslant \eta,
$$

then we shall say that the sequence $\left\{X_{j}, j=1,2,3, \ldots\right\}$ satisfies the condition $L^{\prime}$.

In the case where the $X_{j}$ are identically distributed, the conditions $L$ and $L^{\prime}$ are automatically satisfied. A simple non-identically distributed case in which they are satisfied is that in which some of the $\phi_{j}(t)$ are of the form $f(t) \phi(t)$ where $f(t)$ and $\phi(t)$ are characteristic functions, $f(t)$ being real, while others are of the form $\phi(t)$. We then have

$$
\left|\phi_{j}(t)\right|=|f(t)||\phi(t)| \leqslant|\phi(t)|
$$

or

$$
\left|\phi_{j}(t)\right|=|\phi(t)|
$$

and, similarly,

$$
\left|I_{j}(t)\right|=|f(t)||I(t)| \leqslant|I(t)|
$$

or

$$
\left|I_{j}(t)\right|=|I(t)|
$$

Lemma $1(5,61 ; 7$, Lemma 1$)$. Let $X$ be a non-degenerate random variable with characteristic function $\phi(t)$. There exist constants $\delta>0$ and $c>0$ such that

$$
|\phi(t)| \leqslant 1-c t^{2} \quad \text { for }|t| \leqslant \delta .
$$


Lemma 2. Let $\left\{X_{j}, j=1,2,3, \ldots\right\}$ be a sequence of independent, nondegenerate random variables satisfying the condition L. Write

$$
S_{n}=\sum_{j=1}^{n} X_{j}
$$

$I_{n}$ is an interval on the $x$-axis and $l\left(I_{n}\right)$ is its length. $c$ is a constant that is independent of $n$ and $I_{n}$.

(a) If $l\left(I_{n}\right) \leqslant n^{p}, 0<p<\frac{1}{2}$, then $\operatorname{Pr}\left(S_{n} \in I_{n}\right) \leqslant c / n^{\frac{1}{2}-p}$

(b) If $l\left(I_{n}\right) \leqslant \epsilon \sqrt{ } n, \epsilon>0$, then $\operatorname{Pr}\left(S_{n} \in I_{n}\right) \leqslant \epsilon(c+\xi(\epsilon, n))$ where $\xi(\epsilon, n) \rightarrow 0$ for every fixed $\epsilon>0$.

(c) If $l\left(I_{n}\right) \leqslant M$ (constant), then $\operatorname{Pr}\left(S_{n} \in I_{n}\right) \leqslant c / \sqrt{ } n$.

(d) $\max _{a} \operatorname{Pr}\left(S_{n}=a\right) \leqslant c / \sqrt{ } n$.

These results cannot, in general, be improved.

This lemma is an extension of (7, Theorem 1) from the case of identically distributed random variables to the case of random variables satisfying the condition $L$. Only minor modifications are necessary to the proof given in (7) to make it applicable in this case. It is necessary to replace $\phi^{n}(t)$ by

$$
\prod_{j=1}^{n} \phi_{j}(t)
$$

$\left(\phi_{j}(t)\right.$ being the characteristic function of $\left.X_{j}\right)$. Then, in view of the condition $L$, we have the relation

$$
\prod_{j=1}^{n}\left|\phi_{j}(t)\right| \leqslant|\phi(t)|^{n}
$$

in $|t| \leqslant \delta_{1}$ (say) for some characteristic function $\phi(t)$. Thus, by virtue of Lemma 1,

$$
\begin{aligned}
\int_{|t| \leqslant \delta} \prod_{j=1}^{n}\left|\phi_{j}(t)\right| d t & \leqslant \int_{|t| \leqslant \delta}|\phi(t)|^{n} d t \\
& \leqslant \int_{|t| \leqslant \delta}\left(1-c t^{2}\right)^{n} a t \\
& \leqslant \int_{|t| \leqslant \delta} e^{-c t^{2} n} d t \leqslant c_{1} / \sqrt{ } n
\end{aligned}
$$

here $\delta=\min \left(\delta_{1}, \delta_{2}\right)$ where $\delta_{2}$ is the $\delta$ of Lemma 1. The equation (2.2) of (7) then follows and the remainder of the proof is identical.

Lemma 3 (Gil-Pelaez' inversion formula (3; see also 7, Theorem 2 and 10 )). Suppose the random variable $X$ has distribution function $F(x)$ and characteristic function $\phi(t)$. Then

$$
\begin{array}{r}
\frac{1}{2}[F(x-0)+F(x+0)]=\frac{1}{2}+\lim _{A \rightarrow 0} \lim _{B \rightarrow \infty} \frac{1}{2 \pi i} \int_{A}^{B} t^{-1}\left\{e^{i x t} \phi(-t)-e^{-i x t} \phi(t)\right\} d t \\
=\frac{1}{2}+\lim _{A \rightarrow 0} \frac{1}{2 \pi i} \int_{A}^{\delta} t^{-1}\left\{e^{i x t} \phi(-t)-e^{-i x t} \phi(t)\right\} d t+R(1, x, \delta)
\end{array}
$$


where $\delta>A>0$ and

$$
R(1, x, \delta)=\frac{1}{\pi} \int_{-\infty}^{\infty} d F(y) \int_{\delta}^{\infty} \frac{\sin (x-y) t}{t} d t .
$$

The use of the improper lower limit in the range of integration enables us to remove the restriction

$$
\int_{-\infty}^{\infty} \log (1+|x|) d F(x)<\infty
$$

which was imposed in (7).

Note. The corresponding remainder term for the random variable $S_{n}$ (an $n$-element convolution) will be denoted $R(n, x, \delta)$.

Lemma 4. Suppose the sequence $\left\{X_{j}, j=1,2,3, \ldots\right\}$ of random variables satisfies the condition L. For any $\epsilon, 0<\epsilon<\frac{1}{2}$, there exists a constant c, independent of $n$ and $x$, such that for all $\delta<\delta_{0}$,

$$
|R(n, x, \delta)| \leqslant c n^{\epsilon-\frac{1}{2}}
$$

This lemma is an extension of the lemma of (1) from the case of identically distributed random variables to the case of random variables satisfying the condition $L$. $\delta_{0}$ is the $\delta_{0}$ of Lemma 2. The proof in (1) remains formally unchanged in view of the results of Lemma 2.

\section{Results.}

Theorem 1. Suppose $\left\{X_{j}, j=1,2,3, \ldots\right\}$ is a sequence of independent non-degenerate random variables satisfying the condition $L^{\prime}$. Then for $-\infty<x<\infty, 1 \geqslant \beta>\frac{1}{2}+p$, and $0 \leqslant p<\frac{1}{2}$,

$$
\sum_{n=1}^{\infty} n^{-\beta}\left|\operatorname{Pr}\left(S_{n}<n^{p} x\right)-\frac{1}{2}\right|<\infty
$$

as long as

$$
\lim _{A \rightarrow 0} \int_{A}^{\delta} t^{2 \beta-5}|I(t)| d t<\infty
$$

for some $\delta>0$. $\left(I(t)\right.$ is the uniform bound from the definition of $L^{\prime}$.)

Proof. We shall establish first that when the sequence $\left\{X_{j}, j=1,2,3, \ldots\right\}$ satisfies the condition $L^{\prime}, \beta>\frac{1}{2}$, and

$$
\lim _{A \rightarrow 0} \int_{A}^{\delta} t^{2 \beta-5}|I(t)| d t<\infty
$$

then

$$
\sum_{n=1}^{\infty} n^{-\beta}\left|\operatorname{Pr}\left(S_{n}<0\right)-\frac{1}{2}\right|<\infty
$$


The proof of this result follows along lines similar to the corresponding work in ( 1 and $\mathbf{7})$.

Making use of Lemma 3, we have

$$
\begin{aligned}
\operatorname{Pr}\left(S_{n}<0\right)-\frac{1}{2}= & \frac{1}{2}\left\{F_{n}(0+)-F_{n}(0-)\right\}-\frac{1}{2} \operatorname{Pr}\left(S_{n}=0\right)-\frac{1}{2} \\
= & \frac{1}{2 \pi i} \lim _{A \rightarrow 0} \int_{A}^{\delta} t^{-1}\left\{\prod_{j=1}^{n} \phi_{j}(-t)-\prod_{j=1}^{n} \phi_{j}(t)\right\} d t \\
& \quad+R(n, 0, \delta)-\frac{1}{2} \operatorname{Pr}\left(S_{n}=0\right) \\
= & \frac{1}{2 \pi i} \lim _{A \rightarrow 0} \int_{A}^{\delta} t^{-1} \prod_{j=1}^{n}\left|\phi_{j}(t)\right| \sin \left(\sum_{j=1}^{n} \arg \phi_{j}(t)\right) d t \\
& \quad+R(n, 0, \delta)-\frac{1}{2} \operatorname{Pr}\left(S_{n}=0\right),
\end{aligned}
$$

where $\delta>0$, which is yet to be completely specified, is chosen so small that the inequalities of conditions $L, L^{\prime}$, and Lemma 1 are all satisfied. Therefore, using the condition $L$,

$$
\begin{aligned}
& \sum_{n=1}^{\infty} n^{-\beta}\left|\operatorname{Pr}\left(S_{n}<0\right)-\frac{1}{2}\right| \\
& \leqslant \frac{1}{\pi} \lim _{A \rightarrow 0} \int_{A}^{\delta} t^{-1} \sum_{n=1}^{\infty} n^{-\beta} \prod_{j=1}^{n}\left|\phi_{j}(t)\right| \sum_{j=1}^{n}\left|\arg \phi_{j}(t)\right| d t \\
& \quad+\sum_{n=1}^{\infty} n^{-\beta}|R(n, 0, \delta)|+\frac{1}{2} \sum_{n=1}^{\infty} n^{-\beta} \operatorname{Pr}\left(S_{n}=0\right) \\
& \leqslant \frac{1}{\pi} \lim _{A \rightarrow 0} \int_{A}^{\delta} t^{-1} \sum_{n=1}^{\infty} n^{-\beta}|\phi(t)|^{n} \sum_{j=1}^{n}\left|\arg \phi_{j}(t)\right| d t \\
& \quad+\sum_{n=1}^{\infty} n^{-\beta}|R(n, 0, \delta)|+\frac{1}{2} \sum_{n=1}^{\infty} n^{-\beta} \operatorname{Pr}\left(S_{n}=0\right) .
\end{aligned}
$$

We consider separately the three parts on the right-hand side of (1).

In view of our choice of $\delta$, it follows from Lemma 4 with $\epsilon$ chosen to be less than $\beta-\frac{1}{2}$ that

$$
\sum_{n=1}^{\infty} n^{-\beta}|R(n, 0, \delta)|<\infty .
$$

Also, it follows from Lemma 2, part (d) that

$$
\sum_{n=1}^{\infty} n^{-\beta} \operatorname{Pr}\left(S_{n}=0\right)<\infty .
$$

Now we can write

$$
\phi_{j}(t)=R_{j}(t)+i I_{j}(t)
$$

where $R_{j}(t)$ and $I_{j}(t)$ are real functions, bounded on any finite interval. Then $\arg \phi_{j}(t)=\arctan \left\{I_{j}(t)\left[R_{j}(t)\right]^{-1}\right\}$. 
$R_{j}(t)\left(=\frac{1}{2}\left(\phi_{j}(t)+\phi_{j}(-t)\right)\right.$ is itself a characteristic function and hence is continuous about $R_{j}(0)=1$ in a neighbourhood of the origin. Therefore, given suitably small $\epsilon>0$ we can find $\delta_{j}>0$ so small that $\left|R_{j}(t)-1\right|<\epsilon$ in $|t| \leqslant \delta_{j}$. Choose $\delta_{0}=\min _{j} \delta_{j}$ (clearly $\delta_{0}>0$ ). Then, in view of the relation $\left|R_{j}(t)-1\right|<\epsilon$ uniformly in $j$ for $|t|<\delta_{0}$, we must have

$$
\left|\arg \phi_{j}(t)\right| \leqslant c\left|I_{j}(t)\right| \leqslant c|I(t)|
$$

for $0 \leqslant t \leqslant \delta_{0}$ and some constant $c>0, I(t)$ being the $I$ of condition $L^{\prime}$. At this stage we further restrict the $\delta$ of the theorem to make it less than the above $\delta_{0}$ as well. Then, using (2), we have

$$
\begin{aligned}
\lim _{A \rightarrow 0} \int_{A}^{\delta} t^{-1} \sum_{n=1}^{\infty} n^{-\beta}|\phi(t)|^{n} \sum_{j=1}^{n}\left|\arg \phi_{j}(t)\right| d t \\
\quad \leqslant \lim _{A \rightarrow 0} c \int_{A}^{\delta} t^{-1} \sum_{n=1}^{\infty} n^{1-\beta}|\phi(t)|^{n}|I(t)| d t .
\end{aligned}
$$

By an Abelian theorem of Widder (11, p. 182, Corollary 1a), we see that

$$
\lim _{u \rightarrow 1-}(1-u)^{2-\beta} \sum_{n=1}^{\infty} n^{1-\beta} u^{n}=\text { const. }
$$

and thus, for $0<u<1$, there is a positive constant $c$ such that

$$
\sum_{n=1}^{\infty} n^{1-\beta} u^{n}<c(1-u)^{\beta-2}
$$

Putting $u=|\phi(t)|$ in this inequality, we obtain, for $t \neq 0$,

$$
\sum_{n=1}^{\infty} n^{1-\beta}|\phi(t)|^{n}<c(1-|\phi(t)|)^{\beta-2} .
$$

Then, by (4) and Lemma 1 , there is a positive constant $k$ such that

$$
\lim _{A \rightarrow 0} \int_{A}^{\delta} t^{-1} \sum_{n=1}^{\infty} n^{1-\beta}|\phi(t)|^{n}|I(t)| d t<k \lim _{A \rightarrow 0} \int_{A}^{\delta} t^{2 \beta-5}|I(t)| d t
$$

and the right-hand side of this inequality is finite by hypothesis. This establishes the first part of the theorem.

The remainder of the proof is now easy to complete. We have, for $x \geqslant 0$,

$$
\begin{aligned}
\sum_{n=1}^{\infty} n^{-\beta}\left|\operatorname{Pr}\left(S_{n}<n^{p} x\right)-\frac{1}{2}\right| \leqslant \sum_{n=1}^{\infty} n^{-\beta} \mid \operatorname{Pr}\left(S_{n}<0\right) & -\frac{1}{2} \mid \\
& +\sum_{n=1}^{\infty} n^{-\beta} \operatorname{Pr}\left(\left|S_{n}\right|<n^{p} x\right) .
\end{aligned}
$$

The first series on the right-hand side converges as we have just shown and the second series converges by Lemma 2 (a) as long as $\beta>\frac{1}{2}+p$. This establishes the result in the case $x \geqslant 0$, and the case $x<0$ follows similarly. 
In general Theorem 1 cannot be extended to the case $\beta=\frac{1}{2}+p, 0 \leqslant p<\frac{1}{2}$. Take, for example, each of the $X_{j}$ as normally distributed with zero mean and unit variance. We have, for $x>0$,

$$
\begin{aligned}
n^{-\beta}\left|\operatorname{Pr}\left(S_{n}<n^{p} x\right)-\frac{1}{2}\right| & =n^{-\beta} \operatorname{Pr}\left(0<S_{n}<n^{p} x\right)=\frac{1}{\sqrt{ }(2 \pi) n^{\beta}} \int_{0}^{n^{p}-\frac{1}{x} x} e^{-\frac{1}{2} u^{2}} d u \\
& \sim \frac{x}{\sqrt{ }(2 \pi) n^{\beta-p+\frac{1}{2}}} \sim \frac{x}{\sqrt{ }(2 \pi) n} \quad \text { when } \beta=\frac{1}{2}+p
\end{aligned}
$$

so that the series

$$
\sum_{n=1}^{\infty} n^{-\beta}\left|\operatorname{Pr}\left(S_{n}<n^{p} x\right)-\frac{1}{2}\right|
$$

diverges when $\beta=\frac{1}{2}+p$.

As an immediate corollary of Theorem 1 we obtain:

Corollary 1. Suppose $\left\{X_{j}, j=1,2,3, \ldots\right\}$ is a sequence of independent, non-degenerate, symmetric random variables satisfying the condition $L$. Then for $-\infty<x<\infty, 1 \geqslant \beta>\frac{1}{2}+p$, and $0 \leqslant p<\frac{1}{2}$,

$$
\sum_{n=1}^{\infty} n^{-\beta}\left|\operatorname{Pr}\left(S_{n}<n^{p} x\right)-\frac{1}{2}\right|<\infty .
$$

Even when $x=0$, the result of Corollary 1 need not extend to the case $\beta=\frac{1}{2}$. We have

$$
\left|\operatorname{Pr}\left(S_{n}<0\right)-\frac{1}{2}\right|=\frac{1}{2} \operatorname{Pr}\left(S_{n}=0\right)
$$

and it is easy to find examples where $\operatorname{Pr}\left(S_{n}=0\right) \sim c n^{-\frac{1}{2}}$ for some positive constant $c$ as $n \rightarrow \infty$. This is the case, for instance, when the $X_{j}$ are identically distributed with a finite variance, zero mean, and characteristic function $\phi(t)=E\left(e^{i t X}\right)$ such that $|\phi(t)|=1$ when $t$ is any multiple of $2 \pi$; cf. (9, p. 75).

The following example shows that the condition $L$ restriction in Theorem 1 (and Corollary 1) cannot be relaxed in general. Take $X_{j}$ as normally distributed with zero mean and variance $j^{-(1-2 p)} \log j, j=1,2,3, \ldots$ Then

$$
\phi_{j}(t)=\exp \left(-\frac{1}{2} j^{-(1-2 p)} \log j t^{2}\right)=\left|\phi_{j}(t)\right|
$$

and $\left|\phi_{j}(t)\right| \rightarrow 1$ as $j \rightarrow \infty$ for all $t$ so the condition $L$ is not satisfied. $S_{n}$ is normally distributed with mean zero and variance

$$
\sum_{k=1}^{n} k^{-(1-2 p)} \log k={a_{n}}^{2} \sim n^{2 p} \log n \quad \text { as } n \rightarrow \infty .
$$

We have, taking $x>0$ for convenience,

$$
\begin{aligned}
\operatorname{Pr}\left(S_{n}<n^{p} x\right)-\frac{1}{2} & =\frac{1}{\sqrt{ }(2 \pi) a_{n}} \int_{0}^{n^{p} x} \exp \left(-\frac{1}{2} u^{2} a_{n}^{-2}\right) d u \\
& =(2 \pi)^{-\frac{1}{2}} a_{n}{ }^{-1} n^{p} x\left(\exp -\frac{1}{2} x^{2} a_{n}{ }^{-2} n^{2 p} \theta_{n}{ }^{2}\right), \quad 0<\theta_{n}<1
\end{aligned}
$$


using the mean value theorem. It follows that

$$
\left|\operatorname{Pr}\left(S_{n}<n^{p} x\right)-\frac{1}{2}\right|=O(\log n)^{-\frac{1}{2}} \quad \text { as } n \rightarrow \infty,
$$

so that the results of neither Theorem 1 nor Corollary 1 can hold. The breakdown in this case comes in Lemma 2 (a).

Lemma 5. Suppose the random variable $X$ has distribution function $F(x)$ and $I(t)$ is the imaginary part of its characteristic function. If, whenever

$$
\int_{-\infty}^{\infty}|x| d F(x)<\infty
$$

we have

$$
\int_{-\infty}^{\infty} x d F(x)=0
$$

then for $\gamma>0$,

$$
\int_{0}^{1}|I(t)| t^{-(1+\gamma)} d t<\infty
$$

as long as

$$
\int_{-\infty}^{\infty}|x|^{\gamma} d F(x)<\infty .
$$

This type of result seems to have been obtained first by Hsu (4). Rosén (7) treats the case $\gamma=2$ while Baum and Katz (1) treat the case $2 \leqslant \gamma<3$. The proof is quite simple and will be omitted; whenever

$$
\int_{-\infty}^{\infty}|x| d F(x)<\infty
$$

and hence

$$
\int_{-\infty}^{\infty} x d F(x)=0
$$

we use

$$
I(t)=\int_{-\infty}^{\infty}(\sin x t-x t) d F(x),
$$

and otherwise we use

$$
I(t)=\int_{-\infty}^{\infty} \sin x t d F(x) .
$$

Lemma 5 is directly applicable in Theorem 1 only in the particular case considered by Baum and Katz (1). For in order that it should be applicable, we must have $\gamma=4-2 \beta$ and $1 \geqslant \beta>\frac{1}{2}$ so that $3>\gamma \geqslant 2$. It is not at all 
surprising that this is the best we can do in general. As pointed out previously, we cannot in general obtain convergence up to $\beta=\frac{1}{2}$ no matter how many moments we assume to be finite. On the other hand, if the $X_{j}$ do not possess a finite variance, it is possible to obtain all sorts of pathological behaviour for the probabilities $\operatorname{Pr}\left(S_{n}<0\right)$ despite the restriction $E X_{j}=0$ when $E\left|X_{j}\right|<\infty$; cf. Spitzer (9, pp. 228-230). He treats the case of identically distributed summands possessing zero mean but not a finite variance and shows that it is possible to find cases where the Césaro limit

$$
\lim _{n \rightarrow \infty} n^{-1} \sum_{k=1}^{n} \operatorname{Pr}\left(S_{k}<0\right)
$$

takes on any value $a, 0 \leqslant a \leqslant 1$, and also cases where the Césaro limit fails to exist altogether.

In the particular case where Lemma 5 is applicable in Theorem 1, we obtain:

Theorem 2. Suppose $\left\{X_{j}, j=1,2,3, \ldots\right\}$ is a sequence of independent, non-degenerate random variables satisfying the condition $L^{\prime}$. If there exists a uniform bound $I(t)$ (for the definition of $L^{\prime}$ ) belonging to the characteristic function of a random variable $Y$ for which $E|Y|^{2+\alpha}<\infty, 0 \leqslant \alpha<1$, and $E Y=0$, then for $0 \leqslant p<\frac{1}{2}(1-\alpha)$,

$$
\sum_{n=1}^{\infty} n^{-\left(1-\frac{1}{2} \alpha\right)}\left|\operatorname{Pr}\left(S_{n}<n^{p} x\right)-\frac{1}{2}\right|<\infty .
$$

Other, albeit rather less general, cases where Theorem 1 may be used are not difficult to find. In order to illuminate the situation slightly, let us mention another expression for $I(t)$ (the imaginary part of a characteristic function $\phi(t)$ which corresponds to a distribution function $F(x))$. This is the form

$$
I(t)=t \int_{0+}^{\infty}[1-F(x)-F(-x+0)] \cos t x d x,
$$

which is obtained by integrating $\phi(t)$ by parts. It is clear from (5) that whether an integral of the form

$$
\lim _{A \rightarrow 0} \int_{A}^{\delta} t^{2 \beta-5}|I(t)| d t
$$

is finite or not depends in a critical way upon $K(x)=1-F(x)-F(-x+0)$, which is in a sense a function measuring departure from symmetry. By considering certain classes of functions $K(x)$ it is quite easy to obtain applications of Theorem 1. For example, we could follow Pitman (6) who has studied cases where $K(x)$ has the property that for $\lambda>0$,

$$
\frac{K(\lambda x)}{K(x)} \rightarrow \frac{1}{\lambda^{m}} \quad \text { as } x \rightarrow 0
$$


for some fixed $m \geqslant 0$ and has obtained asymptotic estimates of the behaviour of $I(t)$ as $t \rightarrow 0+$. This is, of course, just what we need in order to be able to apply Theorem 1 . It does not seem appropriate, however, to pursue this rather specialized matter further in the present context.

\section{REFERENCES}

1. L. E. Baum and M. L. Katz, On the influence of moments on the asymptotic distribution of sums of random variables, Ann. Math. Statist., 34 (1963), 1042-1044.

2. A. A. Borovkov, Limit theorems on the distribution of maxima of sums of bounded lattice random variables. I, Theor. Probability Appl., 5 (1960), 125-155.

3. J. Gil-Pelaez, Note on the inversion theorem, Biometrika, 88 (1951), 481-482.

4. P. L. Hsu, Absolute moments and characteristic functions, J. Chinese Math. Soc., 1 (1951), 259-280.

5. E. Lukacz, Characteristic functions (London, 1960).

6. E. J. G. Pitman, Some theorems on characteristic functions of probability distributions, Proc. 4th Berkeley Symp. on Math. Statist. and Prob., 2 (1961), 393-402.

7. B. Rosén, On the asymptotic distribution of sums of independent identically distributed random variables, Ark. Mat., 4 (1962), 323-332.

8. F. Spitzer, A Tauberian theorem and its probability interpretation, Trans. Amer. Math. Soc., 94 (1960), 150-169.

9. - Principles of random walk (New York, 1964).

10. J. G. Wendel, The non-absolute convergence of Gil-Pelaez' inversion integral, Ann. Math. Statist., 32 (1961), 338-339.

11. D. V. Widder, The Laplace transformation (Princeton, 1964).

Michigan State University 